\title{
RESEARCH ON INVERSION MODELS FOR FOREST HEIGHT ESTIMATION USING POLARIMETRIC SAR INTERFEROMETRY
}

\author{
Lamei Zhang*, Baolong Duan, Bin Zou*
}

Dept. of Information Engineering, Harbin Institute of Technology, Harbin, China, 150001

KEY WORDS: PolInSAR, Forest Height Estimation, Inversion Model, RVoG, S-RVoG, RMoG

\begin{abstract}
:
The forest height is an important forest resource information parameter and usually used in biomass estimation. Forest height extraction with PolInSAR is a hot research field of imaging SAR remote sensing. SAR interferometry is a well-established SAR technique to estimate the vertical location of the effective scattering center in each resolution cell through the phase difference in images acquired from spatially separated antennas. The manipulation of PolInSAR has applications ranging from climate monitoring to disaster detection especially when used in forest area, is of particular interest because it is quite sensitive to the location and vertical distribution of vegetation structure components. However, some of the existing methods can't estimate forest height accurately. Here we introduce several available inversion models and compare the precision of some classical inversion approaches using simulated data. By comparing the advantages and disadvantages of these inversion methods, researchers can find better solutions conveniently based on these inversion methods.
\end{abstract}

\section{INTRODUCTION}

Forest height is important information of forest and usually used in modeling of biomass estimation. PolInSAR system based on the coherent combination of radar interferometry and polarimetry has shown great potential for forest height retrieval as it is sensitive to the vertical structure and physical characteristics of the scattering media and is becoming an important technique for forest parameters extraction. Cloude and Papathanassiou studied this kind of problem firstly and defined the polarimetric interferometric coherence coefficient. They pointed out that the forest parameters can be extracted from this coefficient. Traditional phase difference inversion approach such as DEM differencing method, ESPRIT method is limited by the estimated accuracy of the phase center. These defects limit its application to a certain extent. The three-stage inversion algorithm based on random volume over ground (RVoG) model describes the relation of forest height and polarimetric interferometric coherence. This algorithm is quite simple to implement and widely used and a great number of improvement methods have appeared later. $\mathrm{Lu}$ updated the RVoG model to a sloped random volume over ground ( $\mathrm{S}-\mathrm{RVOG}$ ) model which describes the dependence of the complex coherence on forest height, wave extinction and especially the local range terrain slope. Lavalle studied the temporal coherence and proposed the random motion over ground (RMoG) model. This model successfully solved the influence of temporal coherence by introducing a new parameter termed temporal decorrelation factor $\alpha_{t}$. These studies are of great significance to the development of PolInSAR.

Based on the aforementioned research, we introduce several available inversion models and algorithms and compare the precision of some classical inversion approaches using simulated PolInSAR data with $14 \mathrm{~m}$ standard mean height in order to explore and develop a better inversion method.

\section{PHASE DIFFERENCE INVERSION METHOD}

\subsection{DEM Differencing Approach and PD Coherence Optimization}

This method proposed by Cloude and Papathanassiou (Cloude, 1998) simply define forest height as a phase difference between interferogram of the polarization channel dominated by "pure" volume scattering from the forest canopy and that of the polarization channel dominated by "pure" surface scattering from the ground surface without assuming a forest vertical structure reflectivity function. Then forest height is obtained as,

$$
h_{v}=\frac{\arg \left(\gamma_{v}\right)-\arg \left(\gamma_{s}\right)}{k_{z}}
$$

where $k_{z}=\frac{4 \pi \Delta \theta}{\lambda \sin \theta}$ is the effective wave number, $\theta$ is the angle of incidence and $\Delta \theta$ is the apparent angular separation of the baseline from the scattering point, $\gamma_{v}$ is complex coherence corresponding "pure" volume scattering mechanism, $\gamma_{s}$ is complex coherence corresponding "pure" surface scattering mechanism. This method selected HV polarization to obtain $\gamma_{v}$ and $\mathrm{HH}-\mathrm{VV}$ polarization to compute $\gamma_{s}$. Because coherence phase difference between these two polarizations is small, forest height will be significantly underestimated.

In order to improve the accuracy of phase estimation, Tabb (Tabb, 2002) proposed the PD coherence optimization algorithm. This algorithm is to find two polarization states whose complex coherence shows the largest phase difference in complex plane, which can be solved by finding the eigenvectors of Eq.(3) that maximize the cotangent of the phase of the complex coherence shown as Eq.(2).

$$
\cot (\angle \tilde{\gamma})=\frac{\operatorname{Re}\{\tilde{\gamma}\}}{\operatorname{Im}\{\tilde{\gamma}\}}=\frac{\vec{\omega}^{*}\left(\Omega_{12}+\Omega_{12}^{*}\right) \vec{\omega}}{\vec{\omega}^{*}\left[-j\left(\Omega_{12}-\Omega_{12}^{*}\right)\right] \vec{\omega}}
$$

\footnotetext{
* Corresponding author (zzbei@hit.edu.cn)
} 


$$
\left(\hat{\Omega}_{12}+\hat{\Omega}_{12}^{*}\right) \vec{\omega}=-j \lambda\left(\hat{\Omega}_{12}+\hat{\Omega}_{12}^{*}\right) \vec{\omega}
$$

where $\hat{\Omega}_{12}=\Omega_{12} e^{j\left(\frac{\pi}{2}-\angle t r\left(\Omega_{12}\right)\right)}$. The two eigenvectors $\omega_{H}$ and $\omega_{L}$ corresponding to the largest and the smallest eigenvalue can be obtained through eigenvalue analysis. Then the complex coherence $\gamma_{H}$ and $\gamma_{L}$ can be computed by Eq.(13) and the forest height can be obtained by

$$
h_{v}=\frac{\arg \left(\gamma_{H}\right)-\arg \left(\gamma_{L}\right)}{k_{z}}
$$

\subsection{ESPRIT Method}

The ESPRIT algorithm introduced by Yamada (Yamada, 2001) assumes that there are two scattering phase centers corresponding to the canopy scattering mechanism and the ground scattering mechanism respectively and then extract the forest height using the two separated phase centers. Because the interferometry phase depends strongly on the medium under observation (Yamada, 2006), in the case of backscattering in a reciprocal medium, the signals acquired during a polarimetric interferometric measurement can be represented by,

$$
\begin{aligned}
& S_{1}=\left[\begin{array}{lll}
S_{H H}{ }^{1} & \sqrt{2} S_{H V}{ }^{1} & S_{V V}{ }^{1}
\end{array}\right]^{T} \\
& S_{2}=\left[\begin{array}{lll}
S_{H H}{ }^{2} & \sqrt{2}{S_{H V}}^{2} & S_{V V}{ }^{2}
\end{array}\right]^{T}
\end{aligned}
$$

where ${ }^{T}$ denotes the vector transposition. The ESPRIT algorithm define the overall data vector $\vec{x}$ and its correlation matrix as,

$$
R_{x x}=\left\langle\vec{x} \cdot \vec{x}^{* \mathrm{~T}}\right\rangle
$$

with $\vec{x}=\left[\begin{array}{ll}S_{1}^{T} & S_{2}^{T}\end{array}\right]$, where $\vec{x}=\langle\cdot\rangle$ is the ensemble average in the data processing and ${ }^{*}$ represents the complex conjugation. Then we can estimate the number of dominant scatters $d$ by the eigenvalue of $R_{x x}$,which is given as,

$$
\bar{\lambda}_{1} \geq \bar{\lambda}_{2} \geq \cdots \geq \bar{\lambda}_{d} \geq \bar{\lambda}_{6} \geq 0
$$

where $\bar{\lambda}_{i}$ is the eigenvalue of correlation matrix. Using the eigenvectors $\left(e_{1}, e_{2}, \cdots, e_{d}\right)$ corresponding to the dominant eigenvalues, when a $d \times d$ non-singular matrix $\mathrm{C}$ is introduced, the matrices $S, D$ can be expressed as,

$$
\left[e_{1}, e_{2}, \cdots, e_{d}\right]=\left[\begin{array}{c}
E_{1} \\
E_{2}
\end{array}\right]=\left[\begin{array}{c}
S C \\
S D C
\end{array}\right]
$$

Then the following equation can be derived.

$$
F_{1} D F_{1}^{* T}=F_{2} F_{1}^{* T}
$$

From this equation we can see that the diagonal elements of matrix $\mathrm{D}$ can be gained by the complex eigenvalues of $F_{2} F_{1}^{*}$. Therefore, the interferometric phase of the $m$-th scatter is

$$
\phi_{m}=\arg \left(\lambda_{m}^{\prime}\right)
$$

and polarization state of each local scatter can also be estimated by this way. Assuming that $\phi_{1}, \phi_{2}$ represents the phase center of the forest canopy the ground surface respectively. The forest height can be estimated by

$$
h_{v}=\frac{\phi_{1}-\phi_{2}}{k_{z}}
$$

\section{INVERSION METHOD BASED ON PHYSICAL MODEL}

\subsection{RVoG Model and Three-stage Inversion Method}

A fully polarimetric interferometry system measures each resolution element in the scene from two slightly different look angles. Two scattering matrices $\left[S_{1}\right]$ and $\left[S_{2}\right]$ can be obtained and expressed using the Pauli target vector $\vec{k}_{1}$ and $\vec{k}_{2}$. Then a six-dimension complex matrix of PolInSAR can be defined as,

$$
\left[T_{6}\right]=\left\langle\left[\begin{array}{l}
\vec{k}_{1} \\
\vec{k}_{2}
\end{array}\right]\left[\begin{array}{ll}
\vec{k}_{1}^{*} & \vec{k}_{2}^{* T}
\end{array}\right]\right\rangle=\left[\begin{array}{cc}
T_{11} & \Omega_{12} \\
\Omega_{12}^{* T} & T_{22}
\end{array}\right]
$$

The complex polarimetric interferometric coherence can be shown as,

$$
\tilde{\gamma}\left(\vec{\omega}_{1}, \vec{\omega}_{2}\right)=\frac{\vec{\omega}_{1}^{* T} \Omega_{12} \vec{\omega}_{2}^{* T}}{\sqrt{\left(\vec{\omega}_{1}^{* T} T_{11} \vec{\omega}_{1}^{* T}\right)\left(\vec{\omega}_{2}^{* T} T_{22} \vec{\omega}_{2}^{*}\right)}}=\frac{\vec{\omega}^{* T} \Omega \vec{\omega}}{\vec{\omega}^{* T} T \vec{\omega}}
$$

where $T=\left(T_{11}+T_{22}\right) / 2$ and $\vec{\omega}_{1}=\vec{\omega}_{2}=\vec{\omega}$. The latter is a threecomponent unitary complex vector and related to polarization stage.

In the forest observation, both ground and canopy back scattering are contained in the received signals. A most popular model to describe such a scenario is the RVoG scattering model, shown as,

$$
\tilde{\gamma}(\omega)=e^{j \phi_{0}} \frac{\tilde{\gamma}_{v}+m(\vec{\omega})}{1+m(\vec{\omega})}=e^{j \phi_{0}}\left(\tilde{\gamma}_{v}+\frac{m(\vec{\omega})}{1+m(\vec{\omega})}\left(1-\tilde{\gamma}_{v}\right)\right)
$$

where, $m(\vec{\omega})$ denotes the ground-to-volume scattering ratio being of polarization dependent, $\phi_{0}$ is the phase related to the ground and $\tilde{\gamma}_{v}$ represents the complex coherence for the volume alone. The expression of $\tilde{\gamma}_{v}$ is given as,

$$
\gamma_{v}=\frac{2 \sigma\left(\exp \left(2 \sigma h_{v} / \cos \theta+j k_{z} h_{v}\right)-1\right)}{\left(2 \sigma+j k_{z} \cos \theta\right)\left(\exp \left(2 \sigma h_{v} / \cos \theta\right)-1\right)}
$$

which depends on the wave extinction $\sigma$ and forest height $h_{v}$.

Figure 1 shows the correlation coefficient distribution in the complex plane. It can be seen that the complex coherence follows a straight line in the coherence unit circle which intersects the circle at two points. One of the two points corresponds to the underlying topography phase, so this point is called the true ground phase point; the "pure" volume coherence will be furthest away in distance from the true ground phase point along the line. According to this principle, Cloude and Papathanassiou (Cloude, 2003) developed the Three-Stage inversion algorithm as the following:

(1) Least squares straight line fit.

(2) Vegetation bias removal.

(3) Height estimation.

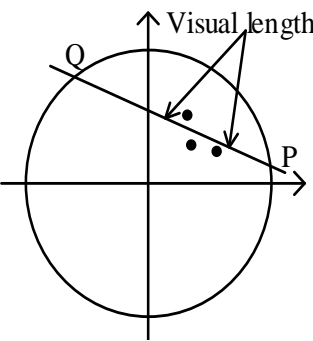

Figure 1. Correlation coefficient distribution image 


\subsection{S-RVoG Model}

The S-RVoG model (Lu, 2013) based on RVoG model describes the dependence of coherence on forest height, average extinction and especially the local range terrain slope. By aligning the reference frame along the local terrain slope and changing the corresponding radar geometrical configuration, a significant model complexity reduction is achieved. This model changes the volume coherence as follow,

$$
\gamma_{v}^{\prime}=\frac{2 \sigma\left(\exp \left(2 \sigma h_{v} / \cos \theta^{\prime}+j k_{z}^{\prime} h_{v}\right) \cos \alpha-1\right)}{\left(2 \sigma+j k_{z}^{\prime} \cos \theta^{\prime}\right)\left(\exp \left(2 \sigma h_{v} / \cos \theta^{\prime} \cos \alpha\right)-1\right)}(16)
$$

where $\theta^{\prime}=\theta-\alpha$ denotes the local incidence angle and $k_{z}^{\prime}=\frac{4 \pi \Delta \theta^{\prime}}{\lambda \sin \theta^{\prime}}$ represents the effective vertical wave number. Obviously, the S-RVoG model is consistent with RVoG model in the case of $\alpha=0$.

\subsection{RMoG Model}

The RMoG model (Lavalle, 2015) which incorporates both volumetric and temporal decorrelation effects combines the volumetric coherence inherited from the RVoG model with the temporal coherence based on Gaussian-statistic motion of the scattering elements. The coherence expression of RMoG model gives as,

$$
\gamma=e^{j \phi_{g}} \frac{\mu \gamma_{t g}+\gamma_{v t} e^{-j \phi_{g}}}{\mu+1}
$$

where, $\mu$ is the ground-to-volume scattering ratio, the temporal coherence of the ground surface $\gamma_{t g}$ and the temporal volumetric coherence of the canopy layer without ground surface $\gamma_{v t}$ are defined by

$$
\begin{gathered}
\gamma_{t g}=\exp \left[-\frac{1}{2}\left(\frac{4 \pi}{\lambda}\right)^{2} \sigma_{g}^{2}\right] \\
\gamma_{v t}=e^{j \phi_{g}} \gamma_{t g} \frac{p_{1}\left[e^{\left(p_{2}+p_{3}\right) h_{v}}-1\right]}{\left(p_{2}+p_{3}\right)\left(e^{p_{1} h_{v}}-1\right)}
\end{gathered}
$$

In the above equations, $\sigma_{g}$ denotes the motion standard deviation of the scatters located effectively at $z=z_{g}, h_{r}$ is the reference height for canopy motion, $\varphi_{g}=k_{z} z_{g}$ is the ground topographic phase and $p_{1}, p_{2}$ and $p_{3}$ are defines as,

$$
\begin{aligned}
& p_{1}=\frac{2 \sigma}{\cos (\theta-\alpha)} \\
& p_{2}=p_{1}+j k_{z} \\
& p_{3}=-\frac{\Delta \sigma^{2}}{2 h_{r}}\left(\frac{4 \pi}{\lambda}\right)^{2}
\end{aligned}
$$

Eq.(17) becomes the RVoG model when the motion parameters are set to zero, $\sigma_{v}=0$ and $\sigma_{g}=0$.

$$
\gamma_{v}=\frac{e^{j \phi_{g}}}{\mu+1}\left[\mu+\frac{p_{1}\left(e^{p_{2} h_{v}}-1\right)}{p_{2}\left(e^{p_{1} h_{v}}-1\right)}\right]
$$

When introducing a new parameter $\alpha_{t}$ named temporal decorrelation factor, the total coherence becomes

$$
\gamma=\gamma_{v} \alpha_{t}
$$

where $\alpha_{t}$ is given as,

$$
\alpha_{t}=\gamma_{t g} p_{2} \frac{\mu\left(p_{2}+p_{3}\right)\left(e^{p_{1} h_{v}}-1\right)+p_{1}\left[e^{\left(p_{2}+p_{3}\right) h_{v}}-1\right]}{\left(p_{2}+p_{3}\right)\left[\mu p_{2}\left(e^{p_{1} h_{v}}-1\right)+p_{1}\left(e^{p_{2} h_{v}}-1\right)\right]}
$$

This equation shows how temporal phenomena affect the volumetric coherence. This is indeed what is commonly referred to as temporal decorrelation.

\section{EXPERIMENT AND DISCUSSION}

\subsection{Data Sets Introduction}

The simulated data from PolSARProSim software is used for this syudy. The center frequency is at $1.3 \mathrm{GHz}$ and the incidence angle is 45 degree. The platform altitude is at $3000 \mathrm{~m}$ above ground with $-6.1 \mathrm{~m}$ vertical baseline and $6.01 \mathrm{~m}$ horizontal baseline, respectively. The data were processed for $1.5 \mathrm{~m}$ range resolution and $1.5 \mathrm{~m}$ resolution in azimuth.

The forest region covers a $0.5 \mathrm{Ha}$ area whose stand density is $300 \mathrm{stem} / \mathrm{Ha}$. The dominant tree species of this area is composed of deciduous and the stand height is $14 \mathrm{~m}$. The top of image corresponds to far range which can be identified due to the shadowing effect at the borders of the forest. The forest scenario considered is the placed above a Bragg surface with slightly sloped terrain. Figure 2 shows the Pauli decomposition image of the simulated data with 129 pixels in range and 111 in azimuth.

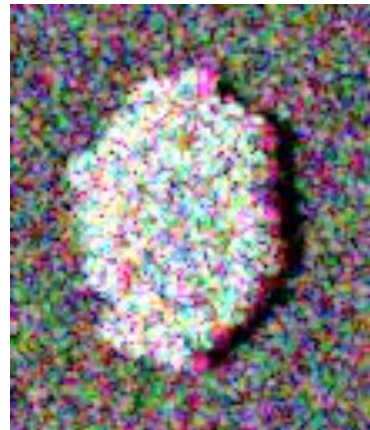

Figure 2. Pauli decomposition image of the simulated data

\subsection{Experiment Result Analysis}

Figure 3 is a plot of the forest height estimation of DEM differencing method in the 52th row of azimuth transect line. It can be seen that the forest height ranges from $0 \mathrm{~m}$ to $5.5 \mathrm{~m}$ with $2.80 \mathrm{~m}$ mean height. Compared with the actual $14 \mathrm{~m}$ tree height, forest height is significantly underestimated because the difference between coherence phase of $\mathrm{HV}$ and $\mathrm{HH}-\mathrm{VV}$ is small.

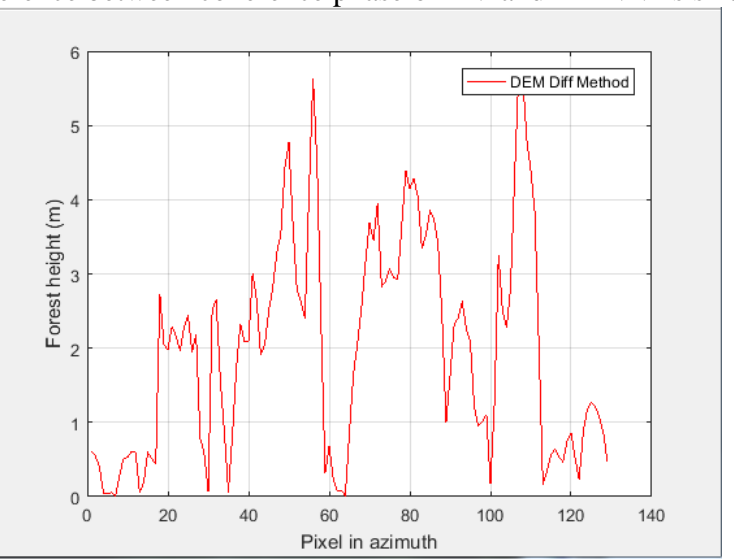

Figure 3. Height estimation result of DEM differencing method 
Forest height for data sets is obtained using DEM differencing method with $\gamma_{H}$ and $\gamma_{L}$ defined by the PD polarimetric interferometric coherence optimization algorithm. Figure 4 shows the result image with forest height ranges from $0 \mathrm{~m}$ to $6.5 \mathrm{~m}$. The mean height is $2.91 \mathrm{~m}$. Compared with DEM differencing method, the result has a certain improvement but still significantly underestimate the forest height. The result indicates the capability for PD coherence optimization algorithm to extract coherent component of "pure" volume scattering and "pure" surface scattering mechanism is limited.

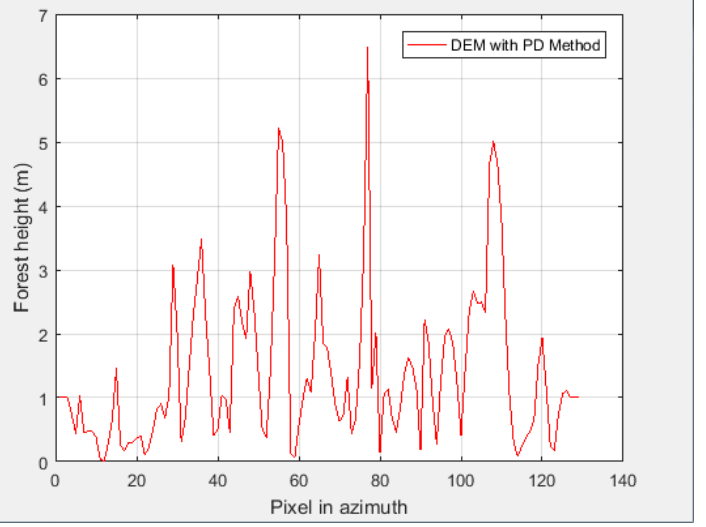

Figure 4. Height estimation result of DEM differencing method with PD coherence optimization

Figure 5 is the height estimation result using ESPRIT method. We can see that tree height ranges $0 \mathrm{~m}$ to $23 \mathrm{~m}$ with $11.08 \mathrm{~m}$ mean height. Compared with the actual $14 \mathrm{~m}$ tree height, there are biased in tree height retrieval results due to the existence of depolarized components.

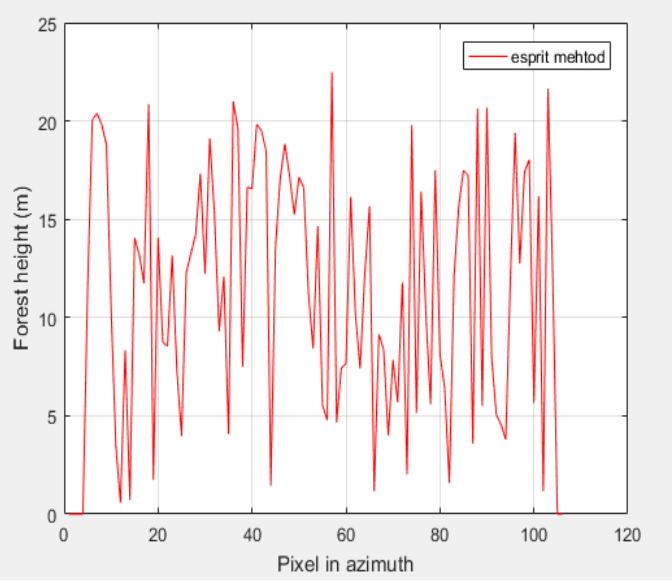

Figure 5. Height estimation result of ESPRIT method

When the terrain slope is set to $20 \%$ with other parameters remain, the results of Three-Stage inversion method using RVoG model and S-RVoG model are shown as Figure 6 and Figure 7, respectively. As the plots confirm, the height of RVoG model ranges from $2 \mathrm{~m}$ to $15 \mathrm{~m}$ and the mean height is $7.95 \mathrm{~m}$. In other side, for $\mathrm{S}-\mathrm{RVoG}$ model, the height ranges from $2 \mathrm{~m}$ to $16 \mathrm{~m}$ with $8.30 \mathrm{~m}$ mean height. The result is improved to a certain extent. It can be seen that a direct application of the RVoG model inversion will yield some errors in the estimation without consideration of the local terrain slope.

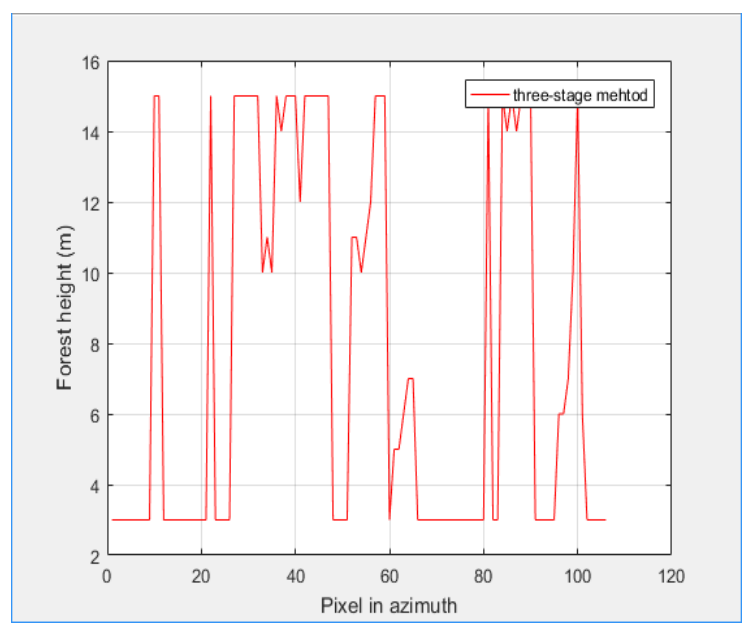

Figure 6. Height estimation result of Three-Stage inversion method using RVoG model

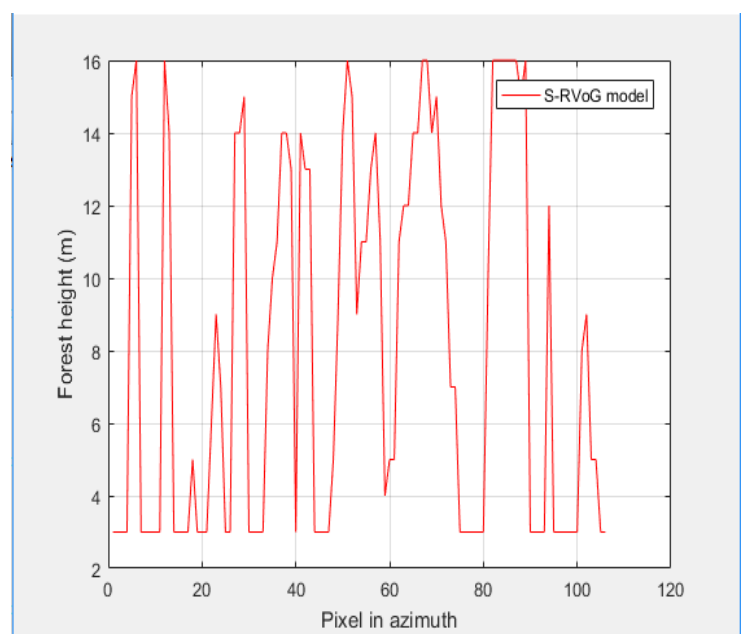

Figure 7. Height estimation result of Three-Stage inversion method using S-RVoG model

The performance comparison of these inversion methods is listed in Table 1. Obviously, these methods all underestimate tree height especially the DEM differencing method. PD coherence optimization algorithm has a certain improvement compared with DEM method. But the result shows that the capability for this algorithm is limited. The S-RVoG model can be able to correct the terrain distortion effectively and provide more accurate estimation of forest height than the RVoG model. But the result still makes the height underestimated. We don't verify the RMoG model, but it still deserves trying because the temporal decorrelation has always been an important influence for the inversion accuracy.

Table 1. Comparison of forest height inversion results

\begin{tabular}{|l|c|}
\hline Inversion method/model & Height $(\mathrm{m})$ \\
\hline Actual height & 14 \\
DEM Differencing Method & 2.80 \\
PD Coherence Optimization & 2.91 \\
ESPRIT Method & 11.08 \\
RVoG Model & 7.95 \\
S-RVoG Model & 8.30 \\
\hline
\end{tabular}




\section{CONCLUSION}

Several available inversion models and algorithms were introduced in this paper. We compared the precision of some classical inversion approaches using simulated PolInSAR data. The experiment results indicate that these methods all underestimate the tree height and still need to improve. In order to explore and develop a better inversion method, further work need to be done.

\section{ACKNOWLEDGEMENTS}

This work was supported in part by the National Natural Science Foundation of China (61401124) and postdoctoral science-research developmental foundation of Heilongjiang province (LBH-Q13069).

\section{REFERENCES}

Cloude, S. R., Papathanassiou, K. P., 1998. Polarimetric SAR interferometry. IEEE Transaction on Geoscience and Remote Sensing, 36(5), pp. 1551-1556.

Cloude, S. R., Papathanassiou, K. P., 2003. Three-stage inversion process for polarimetric SAR interferometry. IEEE Proceedings Radar, Sonar and Navigation, 150(3), pp. 125-134.
Lavalle, M., Hensley, S., 2015. Extraction of Structural and Dynamic Properties of Forests From PolarimetricInterferometric SAR Data Affected by Temporal Decorrelation. IEEE Transactions on Geoscience and Remote Sensing, 53(9), pp. $4752-4767$.

Lu, H., Suo, Z., Guo, R., Bao, Z., 2013. S-RVoG model for forest parameters inversion over underlying topography. Electronics Letters, 49(9), pp. 618-619.

Yamada, H., Yamaguchi, Y., Kim, E., Rodriguez, Boener, W. M., 2001. Polarimetric SAR interferometry for forest analysis based on the ESPRIT algorithm. IEICE Transaction on Electron, 84(12), pp. 1917-2014.

Yamada, H., Yamazaki, M., Yamaguchi, Y., 2006. On scattering model decomposition of PolSAR and its application to ESPRIT-base Pol-InSAR. Proceeding of 6th European Conference on Synthetic Aperture Radar, Dresden, Germany.

Tabb, M., Orrey, J., Flynn, T., Carande, R., 2002. "Phase diversity: a decomposition for vegetation parameter estimation using polarimetric SAR interferometry. Proceedings of 4 th European Synthetic Aperture Radar Conference, Cologne, Germany. 